\title{
The YEATS family member GAS41 interacts with the general transcription factor TFIIF
}

\author{
Sabrina Heisel1', Nunja C Habel1', Nicole Schuetz ${ }^{1}$ Alessia Ruggieri² and Eckart Meese*1
}

\begin{abstract}
Background: In eukaryotes the transcription initiation by RNA polymerase II requires numerous general and regulatory factors including general transcription factors. The general transcription factor TFIIF controls the activity of the RNA polymerase II both at the initiation and elongation stages. The glioma amplified sequence 41 (GAS41) has been associated with TFIIF via its YEATS domain.

Results: Using GST pull-down assays, we demonstrated that GAS41 binds to both, the small subunit (RAP30) and the large subunit (RAP74) of TFIIF in vitro. The in vivo interaction of GAS41 and endogenous RAP30 and RAP74 was confirmed by co-immunoprecipitation. GAS41 binds to two non-overlapping regions of the C-terminus of RAP30. There is also an ionic component to the binding between GAS41 and RAP30. There was no evidence for a direct interaction between GAS41 and TBP or between GAS41 and RNA polymerase II.

Conclusions: Our results demonstrate binding between endogenous GAS41 and the endogenous TFIIF subunits (RAP30 and RAP74). Since we did not find evidence for a binding of GAS41 to TBP or RNA polymerase II, GAS41 seems to preferentially bind to TFIIF. GAS41 that does not contain a DNA-binding domain appears to be a co-factor of TFIIF.
\end{abstract}

\section{Background}

In eukaryotes general transcription factors control the activity of RNA polymerase II during initiation and elongation of mRNA synthesis. The transcription initiation requires the concerted action of a complex of transcription factors including TFIIA, TFIIB, TFIID, TFIIE, TFIIF, and TFIIH [1,2]. Regulation of transcription is likely to be concerted by additional regulating factors, which are distinct from the general transcription factors in that they are dispensable for basal transcription. Cofactors may also be distinct, because some of them do not directly bind DNA [3]. Some cofactors seem to bridge the interaction between gene-specific transcription factors and general transcription factors, whereas others facilitate chromatin remodeling [4].

An improved understanding of transcription requires further elucidation of the RNA polymerase II machinery. Several lines of evidence suggest that glioma amplified sequence 41 (GAS41) is associated with the general transcription factor complex. Originally, we isolated GAS41

* Correspondence: hgemee@uniklinik-saarland.de

1 Department of Human Genetics, Saarland University, 66421 Homburg, Germany

Full list of author information is available at the end of the article from a glioblastoma cell line as a nuclear protein containing a C-terminal alpha-acidic activation domain and an $\mathrm{N}$-terminal YEATS domain [5]. This YEATS domain is conserved in the YEATS family of transcription factors, including human AF9, ENL, and the yeast ANC1/Taf14 protein. All of the YEATS proteins are components of multi-subunit complexes involved in transcription regulation and chromatin remodeling [6]. For instance, Taf14 is a subunit of TFIID and TFIIF, the chromatin remodeling complexes SWI/SNF, RSC and INO80 as well as the histone acetyltransferase complex NuA3 [7,8]. GAS41 that shows homology to the $\mathrm{N}$-terminus of Taf14 interacts with INI1, the human homolog of the SWI/SNF complex component SNF5 [9].

Furthermore, GAS41 is a subunit of the human TIP60 and SCRAP complexes [10,11]. Targeted disruption of the GAS41 gene in chicken indicates that GAS41 is required for RNA transcription. GAS41 is suggested to function at the assembly of general transcription initiation complexes at the nuclear matrix [12]. We asked whether GAS41 is also associated with the human general transcription factor complex. We examined the interaction of GAS41 and TFIIF which is a heteromeric tetramer of RAP30 and RAP74 [13]. 


\section{Results}

Association of GAS41 with the general transcription factor TFIIF

To analyze whether GAS41 interacts with TFIIF, we recombinantly expressed GST-GAS41, purified the fusion protein and confirmed the expression by SDSPAGE. Coomassie staining showed a $\sim 50 \mathrm{kDa}$ signal corresponding to the combined molecular mass of GSTGAS41. Immunoblot analysis with GAS41 antibody also showed a signal of $\sim 50 \mathrm{kDa}$ (Figure 1A). GST pull-down assay was performed with purified GST-GAS41 immobilized on glutathion-sepharose matrix. GST-GAS41 and GST as control were incubated with purified His-RAP30 and His-RAP74. The eluted protein complexes were subjected to immunoblot analysis. Both, His-RAP30 and His-RAP74 were retained by GST-GAS41 but not by GST alone (Figure 1B). To test for binding of GAS41 with endogenous TFIIF, we performed GST-pull-down assays with HeLa nuclear extract as source for general transcription factors. Immunoblotting confirmed binding of GSTGAS41 with both RAP30 and RAP74 (Figure 1C).

\section{Binding of GAS41 to TFIIF}

To independently confirm the interaction of GAS41 and TFIIF, we performed co-immunoprecipitation experiments. We transiently co-transfected cells with HAGAS41 and FLAG-RAP30. Immunoblotting of cell extracts showed co-expression of HA-GAS41 together with FLAG-RAP30 (Figure 2A). Subsequently, cell lysates were incubated either with anti-FLAG-M2 agarose or anti-HA agarose. By FLAG-immunoprecipitation HAGAS41 as well as endogenous GAS41 was efficiently copurified in complex with FLAG-RAP30. Vice versa, by HA-immunoprecipitation FLAG-RAP30 was efficiently co-purified in complex with HA-GAS41 (Figure 2B, C).

Additionally, we performed immunoprecipitation experiments with the endogenous, transcriptionally relevant TFIIF complex. Therefore, nuclear extracts from HeLa cells without overexpression of RAP30 or RAP74 were subjected to immunoprecipitation using GAS41 antibodies. Immunoblot analysis using RAP30 and RAP74 antibodies revealed that endogenous GAS41 coimmunoprecipitates with endogenous TFIIF (Figure 2D).
A

Coomassie IB: a-GAS41

C

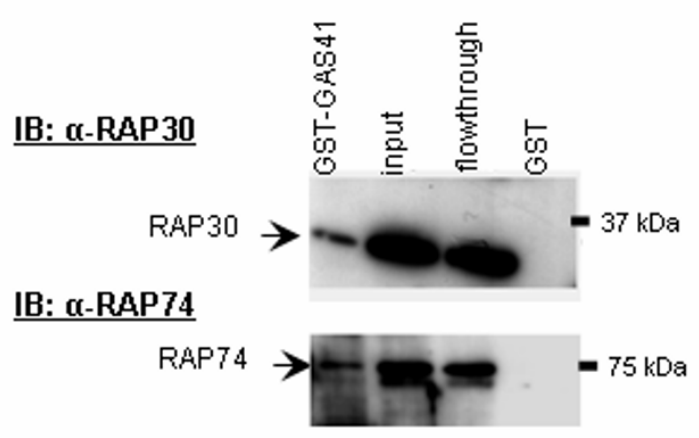

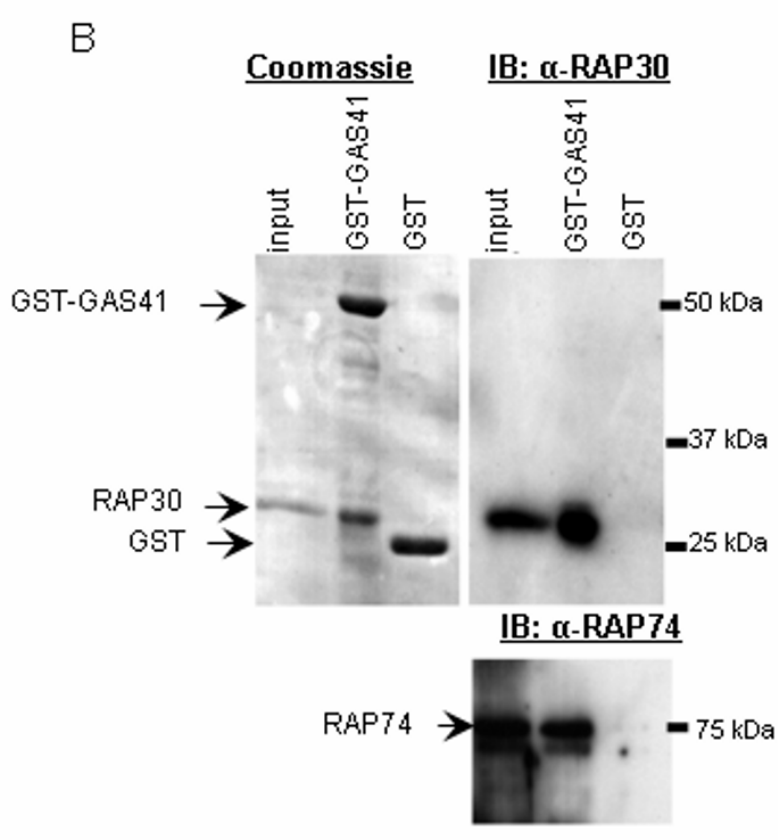

Figure 1 GAS41 directly interacts with TFIIF. (A) GST-GAS41 and GST were purified and subjected to SDS-PAGE. Coomassie staining (left) and immunoblot using GAS41 antibody (right), identified GST-GAS41 and GST. (B) GST pull-down assay with GST-GAS41 as bait retained His-RAP30, while negative control GST did not. His-RAP74 was retained by GST-GAS41 as bait, but not by GST as negative control. (C) GST pull-down assay using HeLa nuclear extract confirms the in vitro interaction of GST-GAS41 with both, RAP30 and RAP74. 


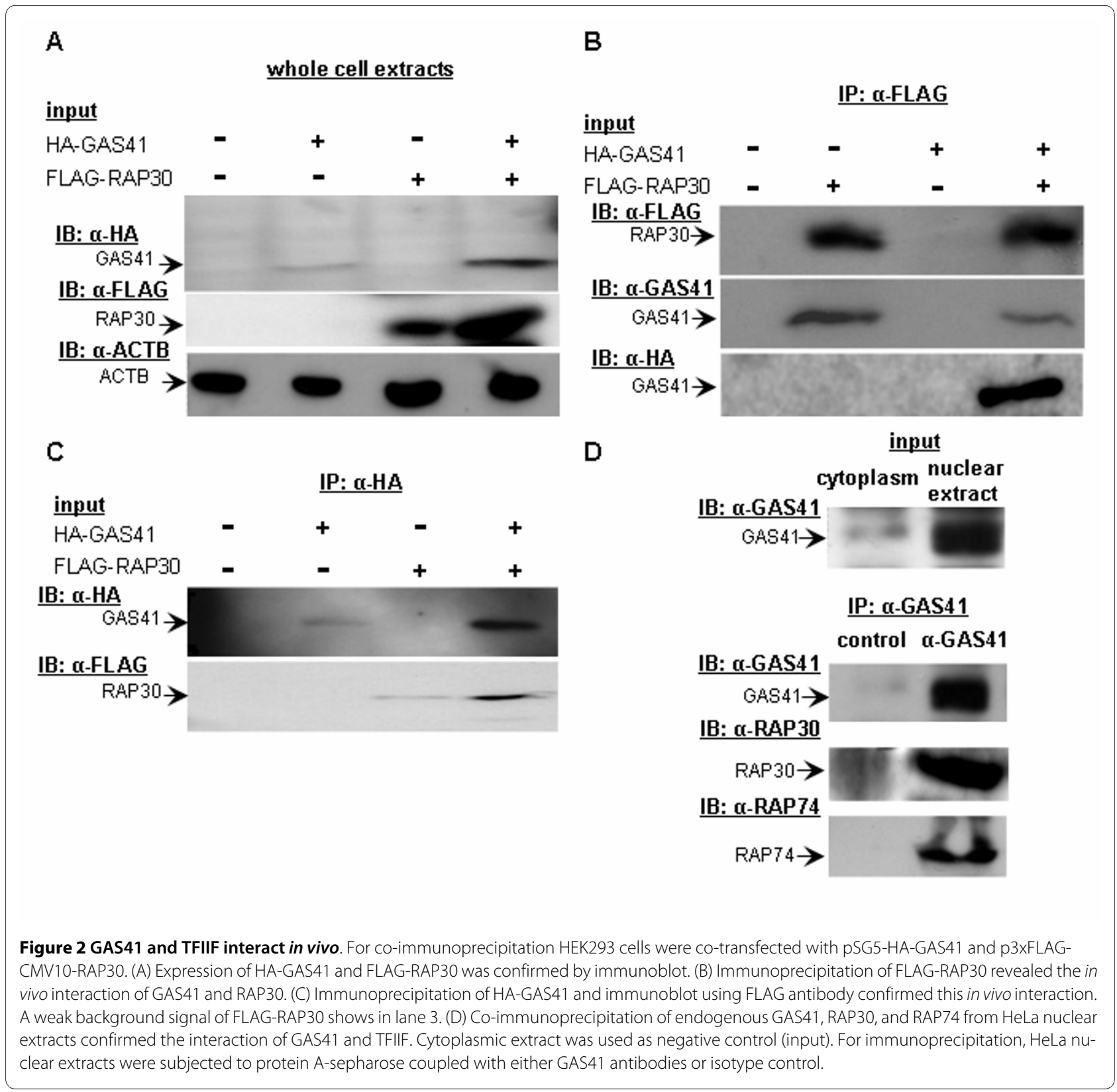

Binding of GAS41 to TFIIF: Specificity and ionic strength We analyzed the direct interaction of GAS41 and the TATA binding protein (TBP) as well as the RNA polymerase II in vivo. Immunoblot analysis revealed no specific binding of TBP to GAS41. Additionally, there is no direct interaction of GAS41 and RNA polymerase II (Figure 3A). These results provide evidence that the GAS41 interaction is specific for this particular general transcription factor.

To examine the stability of protein interactions in the GAS41/TFIIF complex, we analyzed the binding of endogenous RAP30 to the GST-GAS41 fusion protein. GST-GAS41 coupled sepharose was incubated with HeLa nuclear extract. The bound proteins were washed at increasing salt concentrations. Immunoblot analysis showed that $\mathrm{NaCl}$ concentrations of up to $0.4 \mathrm{M}$ cause a slight decrease in GAS41-RAP30 interaction. After washing steps with $0.5 \mathrm{M} \mathrm{NaCl}$, RAP30 is only weakly detectable by immunoblot analysis (Figure 3B). These data indicate an ionic component in the interaction between GAS41 and TFIIF.

\section{Mapping of the GAS41 binding region of RAP30}

To delineate the GAS41 binding region of RAP30, we utilized GST-RAP30 truncation mutants. In detail, we generated fusion proteins representing the RAP74 binding domain (d1), the DNA binding site (d3) and a fragment covering the RAP74 binding domain and the polymerase 
A

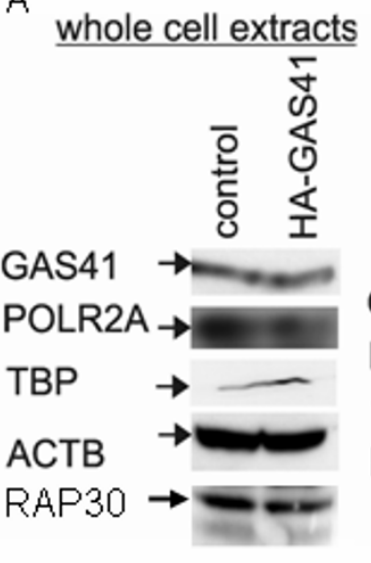

B

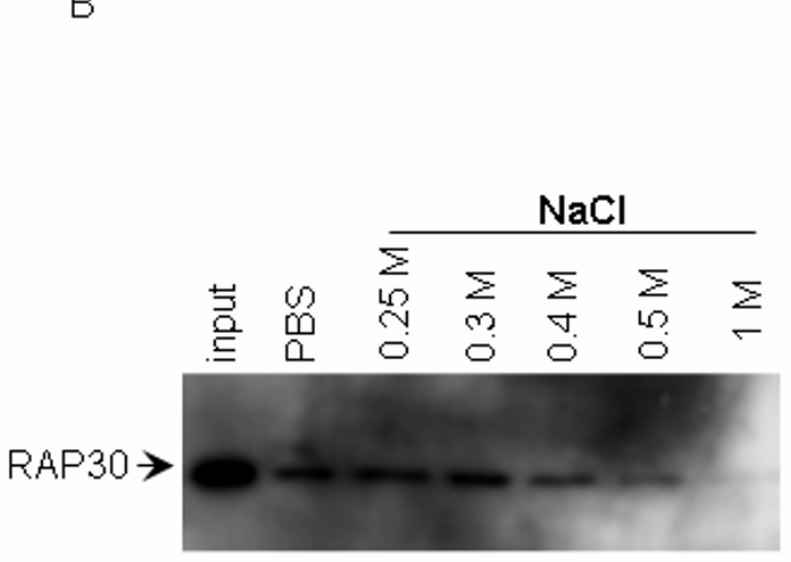

Figure 3 Specific binding of GAS41 to TFIIF. HEK293 cells were transfected with pSG5-HA-GAS41 or pSG5. (A) Expression of HA-GAS41, RNA polymerase II, TBP, RAP30, and ACTB was confirmed by immunoblot. Immunoprecipitation of HA-GAS41 and immunoblot disprove a direct interaction of GAS41 and TBP or RNA polymerase II in vivo. (B) GST-GAS41 was incubated with HeLa nuclear extract as prey and subjected to extensive washing steps with increasing salt concentration. Immunoblot confirmed the interaction of GST-GAS41 and RAP30 up to $0.5 \mathrm{mM} \mathrm{NaCl}$.

binding domain (d2) $[2,13,14]$. GST fusion proteins were subjected to pull-down assays using His-GAS41 as prey. Immunoblot analysis revealed the in vitro interaction of GAS41 with all constructs but d1. These data indicate that GAS41 interacts with two no-overlapping regions of RAP30 (aa101-169 and aa169-249) covering the polymerase and the DNA binding site (Figure 4).

\section{Discussion}

We described GAS41 as a potential transcription factor containing an activation and a YEATS domain $[5,15]$. Here, we report GAS41 as a potential cofactor of TFIIF. TFIIF, consisting of the two subunits RAP30 and RAP74 [16-18], inhibits non-specific transcription initiation by recruiting polymerase II to the pre-initiation complex $[19,20]$. This polymerase II/TFIIF complex attaches through direct interactions of TFIIF with promoter DNA, TFIIB and TFIID [14]. Together with the transcriptional co-activator Mediator, it promotes the association of TFIIE and TFIIH to the pre-initiation complex [21,22].

GST pull-down assays using recombinant RAP30 and RAP74 revealed specific binding of GAS41 to TFIIF in vitro. By using HeLa nuclear extract, we confirmed these results by demonstrating that both subunits of endogenous TFIIF interact with GST-GAS41. Additionally, we verified the interaction with RAP30 and RAP74 in vivo by co-immunoprecipitation. Since GAS41 does not precipitate TBP or RNA polymerase II, GAS41 appears to preferentially interact with TFIIF. Although, the interaction of GAS41 and RAP30 is resistant to high ionic strength, there is an ionic component to the binding between GAS41 and RAP30.
To further characterize the demonstrated interaction of GAS41 and TFIIF, we expressed full length GST-RAP30 as well as three truncated RAP30 mutants. In vitro binding assays revealed direct interaction with all RAP30 constructs except for the RAP74 binding site fragment (d1). We mapped the GAS41 binding site to two non-overlapping regions at the $\mathrm{C}$-terminus of RAP30. Nevertheless, a single GAS41 binding site partly overlapping with the DNA and polymerase binding site can not be excluded. This binding possibly contributes to a function of GAS41 in stabilizing the interaction of TFIIF and DNA.

The function and structure of the RAP30 and RAP74 units have been well defined [14,23-26]. Specifically, RAP30 has been shown to be necessary for pre-initiation complex formation and gene transcription [27-29]. Our data suggest that GAS41 interacts as transcriptional cofactor with the pre-initiation complex via binding to TFIIF. In addition to the recently demonstrated role of GAS41 as co-activator of the sequence-specific transcription factor AP-2 $\beta$ [30] and repressor of the p53 pathway $[31,32]$, the interaction with the pre-initiation complex implies a more general function of GAS41 in cellular transcription. GAS41 is also described as part of TIP60 and SRCAP [32]. Although GAS41 is a component of TIP60, its function in $\mathrm{p} 53$ repression is mediated in a TIP60 independent pathway [32]. Therefore, we assume that GAS41 exerts its function in a mechanism which is independent from the chromatin-modifying complexes TIP60 and SRCAP.

The association of GAS41 to the RAP30 C-terminus possibly contributes to a stabilization of the TFIIF-DNA interaction. Although the functional role of GAS41 in transcription remains to be fully elucidated, we suggest 


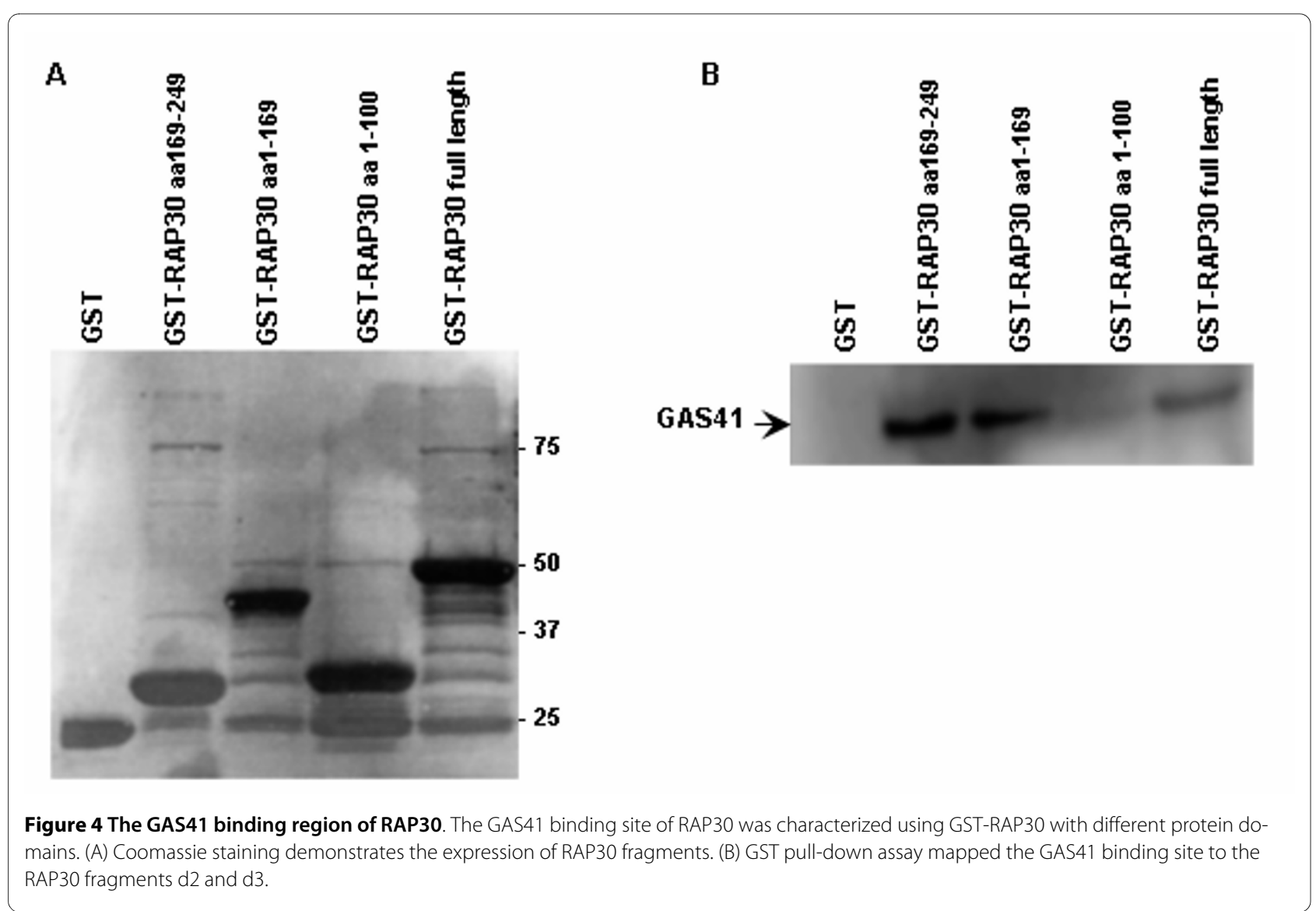

that GAS41 as co-factor of the general transcription complex facilitates transcription initiation and bridges the gap to sequence specific transcription by interaction with appropriate transcription factors.

\section{Conclusion}

We demonstrated that endogenous GAS41 directly interacts with endogenous TFIIF. We mapped the GAS41 binding domain to two regions of RAP30 C-terminus. Without in vivo evidence for a binding of GAS41 to TBP or RNA polymerase II, GAS41 seems to preferentially bind to TFIIF. Without a DNA-binding domain, GAS41 appears to be a co-factor of TFIIF.

\section{Methods}

\section{Expression vectors and antibodies}

Vector pGEX-4T1 (GE Healthcare) was used to express GST-tagged full length GAS41 and RAP30 in BL21 (DE3), as well as RAP30 fragments d1-d3, covering aa1100, aa1-169, and aa169-249. His-GAS41 was expressed in M15 [pRep4] (Qiagen), using the pQE30 vector (Qiagen). The plasmids 6HispET11d-RAP30 and 6HispET11d-RAP74 were a kind gift from R.G. Roeder. Vector pSG5 (Stratagene) was used to express HAGAS41 in HEK293. FLAG-RAP30 was expressed in HEK293 using p3xFLAG-CMV-10 (Sigma). For immuno- blot analysis, we used rabbit RAP30 and RAP74 antibodies (Santa Cruz), rabbit anti-GAS41 [15], mouse-anti TBP (Transduction Laboratories), mouse-anti RNA polymerase II (Millipore), goat anti-GST (GE Healthcare), rat anti-HA (Roche) and mouse anti-FLAG- (Sigma). Peroxidase-conjugated antibodies were from Dianova.

\section{GST pull down assay}

GST fusion proteins were expressed in BL21. After induction with $1 \mathrm{mM}$ isopropyl-D-thiogalactopyranoside (IPTG) $\left(3 \mathrm{~h}, 30^{\circ} \mathrm{C}\right)$, cells were sonicated in PBS containing $1 \%$ TritonX-100. His-RAP30 and-RAP74 were expressed in BL21 (0.4 $\mathrm{mM}$ IPTG, $\left.3 \mathrm{~h}, 30^{\circ} \mathrm{C}\right)$. His-GAS41 was expressed in M15 [pRep4] (0.5 mM IPTG, $\left.2 \mathrm{~h}, 30^{\circ} \mathrm{C}\right)$. Native protein purification by Ni-NTA affinity chromatography was performed according the manufacturer's instruction (Qiagen).

For GST pull-down assay, 10-20 $\mu \mathrm{g}$ GST-fusion protein was coupled to $100 \mu \mathrm{l}$ glutathione-sepharose 4B (GE Healthcare). Matrix was incubated with $250 \mu \mathrm{g}$ HeLa nuclear extract (Promega) or 10-20 $\mu \mathrm{g}$ His-fusion proteins over night at $4^{\circ} \mathrm{C}$. Bound proteins were eluted by boiling in Laemmli buffer, resolved by a $12 \%$ SDS-PAGE and analyzed by Coomassie staining or immunoblot analysis. 
To analyze protein interactions under different salt conditions, GST-GAS41 was incubated with HeLa nuclear extract. Sepharose was washed with $500 \mu \mathrm{l}$ PBS followed by incubation with $500 \mu \mathrm{l}$ PBS containing 0.14-1 $\mathrm{M} \mathrm{NaCl}$, for $45 \mathrm{~min}$ at $4^{\circ} \mathrm{C}$. The remaining proteins were eluted, separated by $12 \%$ SDS-PAGE and subjected to immunoblot.

\section{Cell culture and co-immunoprecipitation}

Cells were grown in DMEM (Invitrogen) supplemented with $10 \%$ fetal calf serum (Biochrom AG) at $37^{\circ} \mathrm{C}$ and $5 \%$ $\mathrm{CO}_{2}$. For immunoprecipitation, $2.5 \times 10^{6}$ cells were cotransfected with pSG5-HA-GAS41 and either p3xFLAGCMV10-RAP30 or p3xFLAG-CMV10. As control pSG5 was co-transfected with p3xFLAG-CMV10-RAP30 or p3xFLAG-CMV10, respectively. Cells were disrupted in lysis buffer $(150 \mathrm{mM} \mathrm{NaCl}, 50 \mathrm{mM}$ Tris/HCl pH 8.0, $1 \%$ NP40, protease inhibitor cocktail (Roche)). For HA-coimmunoprecipitation we used HA-Tag IP/Co-IP kit (Pierce) according to the manufacturer's instructions. For FLAG-co-immunoprecipitation we used FLAG-tagged Protein Immunoprecipitation Kit (Sigma). $250 \mu$ g cell extract was incubated with $30 \mu \mathrm{l}$ FLAG-M2 agarose over night at $4^{\circ} \mathrm{C}$. After washing, bound proteins were eluted using $0.1 \mathrm{M}$ glycine (pH 3.5) and subjected to $15 \%$ SDSPAGE and immunoblot analysis. For nuclear extracts HeLa cells were lysed as described previously [15]. Lysates were subjected to immunoprecipitation using polyclonal GAS41 antibodies [15], rabbit IgG isotype control (Invitrogen), and protein A-sepharose (GE Healthcare). Precipitated protein were eluted by $1 \times$ Laemmli and subjected to 15\% SDS-PAGE and immunoblot analysis.

\section{Authors' contributions}

$\mathrm{SH}, \mathrm{NCH}$ and NS carried out the experiments. AR participated in the design of the study. SH and NCH drafted the manuscript. EM conceived of the study, participated in its design and coordination and drafted the manuscript. All authors read and approved the final manuscript.

\section{Acknowledgements}

This work was supported by a grant from the Deutsche Forschungsgemeinschaft (Fi664/1-1).

\section{Author Details}

'Department of Human Genetics, Saarland University, 66421 Homburg, Germany and 2Department of Infectious Diseases, Molecular Virology, Medical Faculty of Heidelberg, 69120 Heidelberg, Germany

Received: 22 January 2010 Accepted: 12 July 2010

Published: 12 July 2010

\section{References}

1. Thomas MC, Chiang CM: The general transcription machinery and general cofactors. Crit Rev Biochem Mol Biol 2006, 41:105-178.

2. Akashi S, Nagakura S, Yamamoto S, Okuda M, Ohkuma Y, Nishimura Y: Structural characterization of human general transcription factor TFIIF in solution. Protein Sci 2008, 17:389-400.

3. Simons AM, Horwitz AA, Starita LM, Griffin K, Williams RS, Glover JN, Parvin JD: BRCA1 DNA-binding activity is stimulated by BARD1. Cancer Res 2006, 66:2012-2018
4. Naar AM, Lemon BD, Tjian R: Transcriptional coactivator complexes. Annu Rev Biochem 2001, 70:475-501.

5. Fischer U, Heckel D, Michel A, Janka M, Hulsebos T, Meese E: Cloning of a novel transcription factor-like gene amplified in human glioma including astrocytoma grade I. Hum Mol Genet 1997, 6:1817-1822.

6. Schulze JM, Wang AY, Kobor MS: YEATS domain proteins: a diverse family with many links to chromatin modification and transcription. Biochem Cell Biol 2009, 87:65-75.

7. Kabani M, Michot K, Boschiero C, Werner M: Anc1 interacts with the catalytic subunits of the general transcription factors TFIID and TFIIF, the chromatin remodeling complexes RSC and INO80, and the histone acetyltransferase complex NuA3. Biochem Biophys Res Commun 2005, 332:398-403.

8. Cairns BR, Henry NL, Kornberg RD: TFG/TAF30/ANC1, a component of the yeast SWI/SNF complex that is similar to the leukemogenic proteins ENL and AF-9. Mol Cell Biol 1996, 16:3308-3316.

9. Debernardi S, Bassini A, Jones LK, Chaplin T, Linder B, de Bruijn DR, Meese E, Young BD: The MLL fusion partner AF10 binds GAS41, a protein that interacts with the human SWI/SNF complex. Blood 2002, 99:275-281.

10. Doyon Y, Selleck W, Lane WS, Tan S, Cote J: Structural and functional conservation of the NuA4 histone acetyltransferase complex from yeast to humans. Mol Cell Biol 2004, 24:1884-1896.

11. Cai Y, Jin J, Florens L, Swanson SK, Kusch T, Li B, Workman JL, Washburn MP, Conaway RC, Conaway JW: The mammalian YL1 protein is a shared subunit of the TRRAP/TIP60 histone acetyltransferase and SRCAP complexes. J Biol Chem 2005, 280:13665-13670.

12. Zimmermann K, Ahrens K, Matthes S, Buerstedde JM, Stratling WH, Phivan L: Targeted disruption of the GAS41 gene encoding a putative transcription factor indicates that GAS41 is essential for cell viability. J Biol Chem 2002, 277:18626-18631.

13. Wei W, Dorjsuren D, Lin Y, Qin W, Nomura T, Hayashi N, Murakami S: Direct interaction between the subunit RAP30 of transcription factor IIF (TFIIF) and RNA polymerase subunit 5, which contributes to the association between TFIIF and RNA polymerase II. J Biol Chem 2001, 276:12266-12273

14. Fang SM, Burton ZF: RNA polymerase II-associated protein (RAP) 74 binds transcription factor (TF) IIB and blocks TFIIB-RAP30 binding. Biol Chem 1996, 271:11703-11709.

15. Munnia A, Schutz N, Romeike BF, Maldener E, Glass B, Maas R, Nastainczyk W, Feiden W, Fischer U, Meese E: Expression, cellular distribution and protein binding of the glioma amplified sequence (GAS41), a highly conserved putative transcription factor. Oncogene 2001, 20:4853-4863.

16. Conaway JW, Conaway RC: A multisubunit transcription factor essential for accurate initiation by RNA polymerase II. J Biol Chem 1989 264:2357-2362.

17. Conaway RC, Conaway JW: General initiation factors for RNA polymerase II. Annu Rev Biochem 1993, 62:161-190.

18. Flores $\mathrm{O}, \mathrm{Ha}$ I, Reinberg D: Factors involved in specific transcription by mammalian RNA polymerase II. Purification and subunit composition of transcription factor IIF. J Biol Chem 1990, 265:5629-5634

19. Tan S, Garrett KP, Conaway RC, Conaway JW: Cryptic DNA-binding domain in the $C$ terminus of RNA polymerase II general transcription factor RAP30. Proc Natl Acad Sci USA 1994, 91:9808-9812.

20. Wang BQ, Burton ZF: Functional domains of human RAP74 including a masked polymerase binding domain. J Biol Chem 1995, 270:27035-27044.

21. Maxon ME, Goodrich JA, Tjian R: Transcription factor IIE binds preferentially to RNA polymerase Ila and recruits TFIIH: a model for promoter clearance. Genes Dev 1994, 8:515-524.

22. Esnault C, Ghavi-Helm Y, Brun S, Soutourina J, Van Berkum N, Boschiero C, Holstege F, Werner M: Mediator-dependent recruitment of TFIIH modules in preinitiation complex. Mol Cell 2008, 31:337-346.

23. Garrett KP, Serizawa H, Hanley JP, Bradsher JN, Tsuboi A, Arai N, Yokota T, Arai K, Conaway RC, Conaway JW: The carboxyl terminus of RAP30 is similar in sequence to region 4 of bacterial sigma factors and is required for function. J Biol Chem 1992, 267:23942-23949.

24. McCracken S, Greenblatt J: Related RNA polymerase-binding regions in human RAP30/74 and Escherichia coli sigma 70. Science 1991 253:900-902.

25. Sopta M, Burton ZF, Greenblatt J: Structure and associated DNA-helicase activity of a general transcription initiation factor that binds to RNA polymerase II. Nature 1989, 341:410-414. 
26. Yonaha M, Aso T, Kobayashi Y, Vasavada H, Yasukochi Y, Weissman SM Kitajima S: Domain structure of a human general transcription initiation factor, TFIIF. Nucleic Acids Res 1993, 21:273-279.

27. Flores $\mathrm{O}, \mathrm{Lu} \mathrm{H}$, Killeen M, Greenblatt J, Burton ZF, Reinberg D: The small subunit of transcription factor IIF recruits RNA polymerase II into the preinitiation complex. Proc Natl Acad Sci USA 1991, 88:9999-10003.

28. Killeen MT, Greenblatt JF: The general transcription factor RAP30 binds to RNA polymerase II and prevents it from binding nonspecifically to DNA. Mol Cell Biol 1992, 12:30-37.

29. Killeen M, Coulombe B, Greenblatt J: Recombinant TBP, transcription factor IIB, and RAP30 are sufficient for promoter recognition by mammalian RNA polymerase II. J Biol Chem 1992, 267:9463-9466.

30. Ding X, Fan C, Zhou J, Zhong Y, Liu R, Ren K, Hu X, Luo C, Xiao S, Wang Y, Feng D, Zhang J: GAS41 interacts with transcription factor AP-2beta and stimulates AP-2beta-mediated transactivation. Nucleic Acids Res 2006, 34:2570-2578.

31. Llanos S, Efeyan A, Monsech J, Dominguez O, Serrano M: A highthroughput loss-of-function screening identifies novel p53 regulators. Cell Cycle 2006, 5:1880-1885

32. Park JH, Roeder RG: GAS41 is required for repression of the $\mathrm{p} 53$ tumor suppressor pathway during normal cellular proliferation. Mol Cell Biol 2006, 26:4006-4016.

doi: 10.1186/1471-2199-11-53

Cite this article as: Heisel et al., The YEATS family member GAS41 interacts with the general transcription factor TFIIF BMC Molecular Biology 2010, 11:53

Submit your next manuscript to BioMed Central and take full advantage of:

- Convenient online submission

- Thorough peer review

- No space constraints or color figure charges

- Immediate publication on acceptance

- Inclusion in PubMed, CAS, Scopus and Google Scholar

- Research which is freely available for redistribution

Submit your manuscript at www.biomedcentral.com/submit
Ciomed Central 